\title{
Checklist das moscas Asilidae (Diptera) no Mato Grosso do Sul, Brasil
}

\author{
Lucas Araujo Cezar ${ }^{1,2}$
}

\begin{abstract}
1. Museu de Zoologia da Universidade de São Paulo. Avenida Nazaré, 481, Ipiranga, 04263-000, São Paulo, SP, Brasil.
2. Bolsista CNPq. Programa de Pós-Graduação em Entomologia, Faculdade de Filosofia, Ciências e Letras de Ribeirão Preto, Universidade de São Paulo Ribeirão Preto, SP, Brasil. (lucasilidae@gmail.com)
\end{abstract}

Recebido 7 dezembro 2016

Aceito 6 fevereiro 2017

DOI: $10.1590 / 1678-4766 e 2017134$

\begin{abstract}
Checklist of robber-flies (Diptera, Asilidae) in Mato Grosso do Sul state, Brazil. Asilidae comprises over 7,000 species of flies, one of the biggest families in Diptera. They are also called robber flies, due to their predacious behavior. Robber flies are usually more abundant and diverse in dry and open environments. In Mato Grosso do Sul state, Brazil, there are 32 species of robber flies that integrate 17 genera ( $7 \%$ of all described Asilidae species in Brazil). Therefore, Asilidae is a relatively well-known Diptera family for this state. Nevertheless, total Asilidae richness in Mato Grosso do Sul is estimated to be over 80 species.
\end{abstract}

KEYWORDS. Asilinae, Dasypogoninae, Laphriinae, Stenopogoninae, Biota-MS Program.

RESUMO. As moscas Asilidae são insetos predadores, mais abundantes e diversos em ambientes mais secos e de vegetação menos densa. Constituem uma das maiores famílias de Diptera, com mais de 7.000 espécies conhecidas. Estão registradas na literatura 32 espécies de Asilidae que integram 17 gêneros, que ocorrem no estado do Mato Grosso do Sul, o que corresponde a 7\% do conhecido para o Brasil. Isso indica que a família, em comparação com outros dípteros, é bem conhecida no estado. Ainda assim, é possível esperar que a riqueza total de Asilidae passe de 80 espécies no estado.

PALAVRAS-CHAVE. Asilinae, Dasypogoninae, Laphriinae, Stenopogoninae, Programa Biota-MS

Asilidae compreende mais de 500 gêneros e mais de 7.000 espécies distribuídas por quase todos os continentes, com exceção da Antártica, sendo um dos maiores grupos de moscas. A Região Neotropical abriga mais de 1.500 espécies alocadas em 217 gêneros, mas esses números ainda estão distantes de representar a diversidade real do grupo na região. Em território brasileiro já foi registrado quase um terço das espécies conhecidas para a Região Neotropical. É um grupo de diversidade mais significativa em ambientes mais áridos, mesmo presente em grande diversidade de hábitats (CARvalho et al., 2012; PAPAVERo, 2009; Dikow, 2009).

Seus representantes são exclusivamente predadores, tanto na fase adulta quanto na de larva. Os adultos alimentamse por meio do aparelho bucal altamente especializado a uma probóscide em forma de tubo, utilizada para perfurar a presa e injetar saliva com substâncias neurotóxicas. Apresentam olhos altamente desenvolvidos e se caracterizam pelo vértice aprofundado na cabeça. Por sua capacidade de predar insetos, de pequenos mosquitos a grandes libélulas, exercem importante papel ecológico no controle populacional de suas presas (FISHER \& HESPENHEIDE, 1992; FISHER, 2009).

\section{MATERIAL E MÉTODOS}

A lista de espécies que ocorrem no Mato Grosso do Sul foi elaborada tendo como base principal o mais recente catálogo da família para a Região Neotropical (PAPAVERO, 2009). A informação sobre ocorrência das espécies foi refinada a partir de outras referências, citadas na lista. A classificação e nomenclatura dos níveis supragenéricos utilizada segue PAPAVERo (2009), devido à dificuldade de situar os táxons à proposta filogenética recente mais recente (Dikow, 2009), ainda carente de uma chave mais completa.

As localidades com registros estão apresentadas na lista e foram classificadas quanto ao bioma brasileiro em que estão inseridas, segundo limites definidos pelo Instituto Brasileiro do Meio Ambiente e dos Recursos Naturais Renováveis, IBAMA (2012). Por seu caráter mais minucioso, é também apresentada (entre parênteses, apenas em casos conflitantes) a classificação quanto às ecorregiões mundiais disponibilizada pela organização World Wildlife Foundation, WWF (2012).

A lista das espécies de provável ocorrência no Mato Grosso do Sul, mas ainda não registradas, foi inferida a partir das informações de distribuição citadas por PAPAVERO (2009).

\section{RESULTADOS E DISCUSSÃO}

Lista das espécies registradas para o Mato Grosso do Sul. Estão registradas na literatura 32 espécies de 17 gêneros de Asilidae com ocorrência no estado do Mato Grosso do Sul (Tab. I). Esse número corresponde a 7\% das espécies 
Tab. I. Lista de espécies de Asilidae registradas no Mato Grosso do Sul, Brasil. (Biomas: Ce, Cerrado; Ch, Chaco; MA, Mata Atlântica; MS, Matas Secas; Pa, Pantanal). Entre parênteses é apresentada a classificação em ecorregiões segundo a WWF (2012), quando divergente da classificação dos Biomas brasileiros (IBAMA, 2012).

\begin{tabular}{|c|c|c|c|}
\hline Espécie & Localidade & Bioma & Referência \\
\hline \multicolumn{4}{|l|}{ ASILINAE } \\
\hline \multicolumn{4}{|l|}{ Apocleini } \\
\hline Lecania sp. & Maracaju, Três Lagoas & $\mathrm{Ce}, \mathrm{MA}(\mathrm{Ce})$ & Artigas \& Papavero, 1995 \\
\hline Mallophora argentipes Macquart, 1838 & Três Lagoas & $\mathrm{MA}(\mathrm{Ce})$ & ARtigas \& ANGUlo, 1980 \\
\hline Mallophora atra (Macquart, 1834) & Maracaju, Porto Murtinho & $\mathrm{Ce}, \mathrm{Pa}(\mathrm{Ch})$ & ARTIGAS \& ANGULO, 1980 \\
\hline Mallophora calida (Fabricius, 1787) & Maracaju & $\mathrm{Ce}$ & ARTIGAS \& ANGULO, 1980 \\
\hline Mallophora inca (Curran, 1941) & Maracaju & $\mathrm{Ce}$ & ARTigas \& ANGUlo, 1980 \\
\hline Mallophora leschenaulti (Macquart, 1838) & Maracaju & $\mathrm{Ce}$ & ARTIGAS \& ANGULO, 1980 \\
\hline Mallophora lugubris (Lynch Arribálzaga, 1880) & Maracaju, Porto Murtinho & $\mathrm{Ce}, \mathrm{Pa}(\mathrm{Ch})$ & ARTIGAS \& ANGULO, 1980 \\
\hline Mallophora robusta (Wiedemann, 1828) & Maracaju & $\mathrm{Ce}$ & ARTIGAS \& ANGULO, 1980 \\
\hline \multicolumn{4}{|l|}{ DASYPOGONINAE } \\
\hline \multicolumn{4}{|l|}{ Blepharepiini } \\
\hline Blepharepium cajennense coarctatum (Perty, 1833) & Bodoquena, Maracaju & $\mathrm{Ce}, \mathrm{Ce}$ & CARrERA, 1949 \\
\hline Caenarolia anomala (Carrera, 1947) & Porto Murtinho & $\mathrm{Pa}(\mathrm{Ch})$ & CARRERA, 1947 \\
\hline Caenarolia basalis (Curran, 1935) & Porto Murtinho & $\mathrm{Pa}(\mathrm{Ch})$ & CARrera, 1949 \\
\hline Diogmites ferrugineus (Lynch Arribálzaga, 1880) & Salobra & $\mathrm{Pa}(\mathrm{Ce})$ & CARrERA, 1949 \\
\hline \multicolumn{4}{|l|}{ Lastauracini } \\
\hline Lastaurina ardens (Wiedemann, 1828) & Porto Murtinho & $\mathrm{Pa}(\mathrm{Ch})$ & CARrERA, 1945 \\
\hline \multicolumn{4}{|l|}{ Megapodini } \\
\hline Cyrtophrys lynchii (Brèthes, 1901) & Maracaju & $\mathrm{Ce}$ & CARrERA, 1949 \\
\hline Megapoda labiata (Fabricius, 1805) & Maracaju & $\mathrm{Ce}$ & CARRERA, 1949 \\
\hline \multicolumn{4}{|l|}{ Posicionamento incerto } \\
\hline Diogmites vulgaris Carrera, 1947 & São Domingos & $\mathrm{Ce}$ & CARRERA, 1953 \\
\hline \multicolumn{4}{|l|}{ LAPHRIINAE } \\
\hline \multicolumn{4}{|l|}{ Andrenosomatini } \\
\hline Pilica funebris Artigas et al., 1988 & Maracaju & $\mathrm{Ce}$ & ARTIGAS et al.,1988 \\
\hline Pilica pyrrhopyga (Wiedemann, 1828) & Maracaju & $\mathrm{Ce}$ & ARTIGAS et al.,1988 \\
\hline \multicolumn{4}{|l|}{ Atomosiini } \\
\hline Atomosia coxalis Curran, 1930 & Corumbá & $\mathrm{Pa}(\mathrm{MS})$ & Curran, 1930; Papavero, 2009 \\
\hline Atomosia lineata (Curran, 1930) & Corumbá & $\mathrm{Pa}(\mathrm{MS})$ & Curran, 1930; Papavero, 2009 \\
\hline Atoniomyia fulvipes Carrera, 1946 & Porto Murtinho, Salobra & $\mathrm{Pa}(\mathrm{Ch}), \mathrm{Pa}(\mathrm{Ce})$ & Carrera, 1946; Papavero, 2009 \\
\hline Atoniomyia hispidella (Hermann, 1912) & Maracaju & $\mathrm{Ce}$ & CARRERA, 1946 \\
\hline \multicolumn{4}{|l|}{ Posicionamento incerto } \\
\hline Laphygmolestes flavipes Hull, 1962 & Corumbá & $\mathrm{Pa}(\mathrm{MS})$ & PapaVERo, 2009 \\
\hline Protometer bokermanni Artigas et al., 1997 & São Domingos & $\mathrm{Ce}$ & PapaVero, 2009 \\
\hline \multicolumn{4}{|l|}{ Acronychini } \\
\hline Acronyches imitator Hermann, 1921 & Dourados & MA & PAPAVERo, 2009 \\
\hline \multicolumn{4}{|l|}{ Leptogastrini } \\
\hline Schildia fragilis (Carrera, 1944) & Maracaju & $\mathrm{Ce}$ & $\begin{array}{l}\text { PAPAVERO, 2009; } \\
\text { DiKOW \& BAYLESS, } 2009\end{array}$ \\
\hline \multicolumn{4}{|l|}{ Ommatiini } \\
\hline Ommatius costatus Rondani, 1850 & Maracaju & $\mathrm{Ce}$ & VIEIRA, 2009 \\
\hline Ommatius norma (Curran, 1928) & Maracaju & $\mathrm{Ce}$ & SCARBROUGH, 2008 \\
\hline Ommatius orenoquensis (Bigot, 1876) & Três Lagoas & $\mathrm{MA}(\mathrm{Ce})$ & VIEIRA, 2009 \\
\hline Ommatius pulcher (Engel, 1885) & Maracaju, Três Lagoas & $\mathrm{Ce}, \mathrm{MA}(\mathrm{Ce})$ & VIEIRA, 2009 \\
\hline Ommatius spatulatus (Curran, 1928) & Maracaju & $\mathrm{Ce}$ & VIEIRA, 2009 \\
\hline \multicolumn{4}{|l|}{ STENOPOGONINAE } \\
\hline \multicolumn{4}{|l|}{ Enigmomorphini } \\
\hline Dicranus rutilus (Wiedemann, 1821) & Maracaju & $\mathrm{Ce}$ & CARRERA, 1955 \\
\hline
\end{tabular}

conhecidas para o Brasil. Em um inventário realizado para as espécies da Bahia, foram registradas a priori, a partir de dados da literatura, 18 espécies de Asilidae. Após coletas realizadas e exame de material em coleções, esse número subiu para 69 espécies, um acréscimo de quase 300\% (VIEIRA et al., 2006). Ainda que o resultado desse levantamento prévio para o Mato Grosso do Sul tenha recuperado mais espécies que no estudo na Bahia, com base na literatura, é certo que o grupo está subamostrado no estado. Isso pode ser observado na Tabela I; todos os registros se restringem 
a oito localidades apenas, e se concentram em três delas: Maracajú, Porto Murtinho e Três Lagoas.

O Cerrado abriga a grande maioria dos táxons registrada no Mato Grosso do Sul, 22 espécies, sendo 17 exclusivas desse bioma. O Pantanal apresenta nove espécies, sendo que, destas, sete ocorrem apenas nesse bioma. A Mata Atlântica do Mato Grosso do Sul tem a menor riqueza entre os biomas do estado (cinco espécies, sendo três exclusivas). Apesar de o resultado ser compatível com o que se conhece da biologia do grupo, e também com a extensão dos biomas no Mato Grosso do Sul, os eventos de coleta são insuficientes para se afirmar que isso reflita a riqueza real de espécies no estado. Além disso, FisHer (2009) sugeriu que a diversidade de Asilidae em ambientes florestais em geral esteja bastante subestimada, devido à maior dificuldade de se coletar representantes da família nesses ambientes.

Lacunas de conhecimento. Como na maioria dos grupos de invertebrados, a região Centro-Oeste como um todo é bastante subamostrada. Para Asilidae em especial, a diversidade em ambientes florestais deve receber maior atenção nos inventários e estudos taxonômicos, devido à maior dificuldade de coletar espécimes da família nesses ambientes.

Grupos de pesquisa em Asilidae. A família é relativamente bem estudada no Brasil em comparação com outras famílias de Diptera, pois tem sido objeto de estudo de pesquisadores brasileiros desde a primeira metade do século XX. Na década de 1940, o dipterista Messias Carrera, contratado junto ao então Departamento de Zoologia da Secretaria de Agricultura do Estado de São Paulo (posteriormente, Museu de Zoologia), publicou seus primeiros trabalhos sobre a taxonomia de Asilidae. Suas contribuições tratavam principalmente da fauna brasileira e sua produção se estendeu até o fim da década de 1960; além disso, formou outros entomólogos, com destaque para Nelson Papavero, que seguiu os estudos em Diptera, e dedicou grande parte de seu esforço para Asilidae.

Atualmente há três principais núcleos de estudo com sistemática de Asilidae no Brasil (pesquisadores entre parênteses): no Instituto Nacional de Pesquisas da Amazônia, em Manaus (José Albertino Rafael, Rodrigo Marques Vieira e Edgar Alvim); no Museu de Zoologia - USP, em São Paulo (Carlos José Einicker Lamas, Nelson Papavero e Lucas Araujo Cezar); e na Universidade Estadual de Feira de Santana, na Bahia (Freddy Bravo e Ivan Farias Castro).

Principais acervos. As coleções mais representativas de Asilidae no Brasil, segundo CARvalHo et al. (2002) são, em ordem decrescente de quantidade de espécimes montados: Museu de Zoologia da Universidade de São Paulo (MZUSP); Museu Nacional, UFRJ (MNRJ); Departamento de Zoologia da Universidade Federal do Paraná (DZUP) e Instituto Nacional de Pesquisas da Amazônia (INPA).

Perspectivas. Estão listadas abaixo 27 espécies com distribuição registrada no entorno do Mato Grosso do Sul (PAPAVERO, 2009), mas até o momento não registradas no estado: Asilinae [Amblyonychus substitulus (Walker, 1851), Eccritosia barbata (Fabricius, 1787), Eicherax macularis
(Wiedemann, 1821), Mallophora barbipes (Wiedemann, 1819), Mallophora dureti Artigas \& Angulo, 1980, Mallophora emiliae Carrera, 1960, Mallophora nigritarsis (Fabricius, 1805), Mallophora ruficauda (Wiedemann, 1828), Mallophora sylveirii Macquart, 1838, Mallophora zita Curran, 1941, Taurhynchus rubricornis (Macquart, 1838), Wygodasilus ulchripes (Bromley, 1928)]; Dasypogoninae [Amorimius bicolor (Carrera, 1949), Cleptomyia tripartita (Walker, 1854), Diogmites lindigii (Schiner, 1868), Pseudorus distendens (Wiedemann, 1828), Senobasis lenkoi Papavero, 1975)]; Laphriinae [Aphestia annulipes (Macquart, 1838), Cerotainia lynchii (Williston, 1889)]; Ommatiinae [Ommatius arginellus (Fabricius, 1781)]; Stenopogoninae [Archilestris capnoptera (Wiedemann, 1828), Cylicomera rubrofasciata Lynch Arribálzaga, 1881, Dicranus schrottkyi Bezzi, 1910, Enigmomorphus paradoxus Hermann, 1912, Plesiomma caedens (Wiedemann, 1828), Prolepsis fenestrata (Macquart, 1838)]; Stichopogoninae [Townsendia fiebrigii Bezzi, 1909].

Espera-se, portanto, um acréscimo significativo nessa riqueza em um futuro próximo, talvez equivalente ao observado para a Bahia por VIEIRA et al. (2006), como resultado de mais inventários, estudos taxonômicos e da formação de mais taxonomistas. O projeto "Rede temática para estudos de diversidade, sistemática e limites distribucionais de Diptera nos estados do Mato Grosso, Mato Grosso do Sul e Rondônia" (SISBIOTA - CNPq/FAPESP), iniciado em fevereiro de 2010 e ainda em andamento, pode contribuir em um futuro próximo com essa expectativa, pois conta com amostragem em localidades estratégicas nos três estados, além de recurso para formação de mais especialistas na ordem Diptera.

Agradecimentos. A Fundação de Apoio ao Desenvolvimento do Ensino, Ciências e Tecnologia do Estado de Mato Grosso do Sul (Fundect) e a Superintendência de Ciências e Tecnologia do Estado de Mato Grosso do Sul (Sucitec/MS) pelo convite de participação neste fascículo especial da Iheringia, Série Zoologia e o suporte financeiro para sua publicação. À Jéssica Gillung pela leitura crítica do manuscrito. Ao Conselho Nacional de Desenvolvimento Científico e Tecnológico (CNPq) (Proc. n 563256/20109) e Fundação de Amparo a Pesquisa do Estado de São Paulo (FAPESP) (Proc. No. 2010/52314-0) pelo auxílio conferido ao projeto "Rede temática para estudos de diversidade, sistemática e limites distribucionais de Diptera nos estados do Mato Grosso, Mato Grosso do Sul e Rondônia" e bolsa de doutorado conferida ao autor (CNPq, Proc. $n^{\circ}$ 143183/2011-7), aprovados no âmbito do edital SISBIOTA.

\section{REFERÊNCIAS BIBLIOGRÁFICAS}

Artigas, J. N. \& Angulo, A. O. 1980. Revisión del genero Mallophora Macquart por Sistematica Alfa y Taxonomia Numerica (Diptera Asilidae). Gayana, Zoologia 43:5-182.

Artigas, J. N. \& Papavero, N. 1995. The American genera of Asilidae (Diptera): Keys for identification with an atlas of female spermathecae and other morphological details. IX.10. Subfamily Asilinae Leach Lecania-group, with a catalogue of the neotropical species. Theoria 4:33-56.

Artigas, J. N.; Papavero, N. \& Pimentel, T. 1988. The American genera of Asilidae (Diptera): Keys for identification with an atlas of female spermathecae and other morphological details. IV. Key to the genera of Laphriinae Macquart (except tribe Atomosiini Hermann), with the descriptions of three new tribes and five new species. Boletim do Museu Paraense Emilio Goeldi, Série Zoologia 4:211-256. 
Carrera, M. 1945. Relação de alguns Asilidae (Diptera) com suas presas. Papéis Avulsos do Departamento de Zoologia 5:159-166.

CArrera, M. 1946. Sobre algumas espécies do gênero Atoniomyia Hermann, 1912 (Diptera, Asilidae). Papéis Avulsos do Departamento de Zoologia 7:113-128.

Carrera, M. 1947. Asilídeos coligidos no Paraguai pela missão científica brasileira (Diptera). Papéis Avulsos do Departamento de Zoologia 8:39-48.

CARrera, M. 1949. Contribuição ao conhecimento dos Asilidae Neotropicais (Diptera) - I - Sobre as espécies brasileiras com esporão na tíbia. Arquivos de Zoologia do Estado de São Paulo 7:1-148.

CArrera, M. 1953. As espécies neotrópicas do gênero Diogmites (Diptera, Asilidae). Arquivos de Zoologia do Estado de São Paulo 8:169-208.

Carrera, M. 1955. Sobre o gênero Dicranus Loew, 1851 (Diptera, Asilidae). Papéis Avulsos do Departamento de Zoologia 12:235-246.

Carvalho, C. J. B.; Couri, M. S.; Toma, R.; Rafael, J. A.; Harada, A. Y.; Bonatto, S. R.; Henriques, A. L. \& Gastal, H. A. de O. 2002. Principais coleções brasileiras de Diptera: Histórico e situação atual. Monografías Tercer Milenio 2:37-52.

Carvalho, C. J. B.; Rafael, J. A.; Couri, M. S. \& Silva, V. C. 2012. Diptera. In: Rafael, J.; Melo, G. A. R.; Carvalho, C. J. B. De; Casari, S. A. \& Constantino, R. eds. Insetos do Brasil. Diversidade e Taxonomia. Ribeirão Preto, Holos, p.701-743.

Curran, C. H. 1930. New American Asilidae (Diptera). American Museum Novitates 425:1-22.

Dikow, T. 2009. Phylogeny of Asilidae inferred from morphological characters of imagines (Insecta: Diptera: Brachycera: Asiloidea). Bulletin of the American Museum of Natural History 9:165-188.

Dikow, T. \& BAYLESS, K. M. 2009. Taxonomic revision of the genus Schildia Aldrich, 1923 (Diptera: Asilidae: Leptogastrinae) with the description of new extant and extinct species. Insect Systematics \& Evolution 40:253-289.
Fisher, E. M. 2009. Asilidae (robber flies, assassin flies, moscas cazadoras, moscas ladronas). In: Brown, B.V.; Borkent, A.; Cumming, J. M.; Wood, D. M.; Woodley, N. E. \& Zumbado, M. A. eds. Manual of Central American Diptera. Ottawa, NRC Research Press. vol. 1, p.585-632.

Fisher, E. M. \& Hespenheide, H. A. 1992. Taxonomy and biology of Central American robber flies with an illustrated key to genera (Diptera: Asilidae). In: Quintero, D. Q. \& Aiello, A. eds. Insects of Panama and Mesoamerica. Selected studies. New York, Oxford University Press. p.611-632.

IBAMA. Temas vetoriais no formato Shapefile - BR_BIOMAS_IBGE. Disponível em: <http://siscom.ibama.gov.br/shapes/>. Acessado em 20 de agosto de 2012.

PApaVEro, N. 1971. Neotropical Acronyches (Diptera, Asilidae), new or otherwise. Papéis Avulsos de Zoologia 23:145-151.

PApaVEro, N. 2009. Catalogue of Neotropical Diptera. Asilidae. Neotropical Diptera 17:1-179.

Scarbrough, A. G. 2008. New Ommatius Wiedemann from the Americas with two new species groups, keys, and taxonomic notes (Diptera: Asilidae). Insecta Mundi 32:1-14.

VIEIRA, R. M. 2009. Taxonomia de Ommatius Wiedemann, 1821 (Diptera, Asilidae, Ommatiinae) no Brasil. Dissertação de Mestrado. Manaus, Instituto Nacional de Pesquisas da Amazônia, Universidade Federal do Amazonas.

Vieira, R. M.; Castro, I.; Almeida, D.; Alvim, E. \& Bravo, F. 2006. Asilidae (Diptera) da Bahia, Brasil: Sinopse das espécies e chave de identificação. Sitientibus, Série Ciências Biológicas 6:243-256.

WWF. 2012. WWF Science - Data Download. Disponível em: <http:// www.worldwildlife.org/science/data/item1872.html>. Acessado em 13 de março de 2012. 